\title{
Spin generation away from boundaries by nonlinear transport
}

\section{Citation}

Finkler, Ilya G., Hans-Andreas Engel, Emmanuel I. Rashba, and Bertrand I. Halperin. 2007. “Spin Generation Away from Boundaries by Nonlinear Transport." Physical Review B 75 (24) (June 12). doi:10.1103/physrevb.75.241202.

\section{Published Version}

doi:10.1103/physrevb.75.241202

\section{Permanent link}

http://nrs.harvard.edu/urn-3:HUL.InstRepos:24820080

\section{Terms of Use}

This article was downloaded from Harvard University's DASH repository, and is made available under the terms and conditions applicable to Other Posted Material, as set forth at http:// nrs.harvard.edu/urn-3:HUL.InstRepos:dash.current.terms-of-use\#LAA

\section{Share Your Story}

The Harvard community has made this article openly available.

Please share how this access benefits you. Submit a story.

\section{Accessibility}




\title{
Spin generation away from boundaries by nonlinear transport
}

\author{
Ilya G. Finkler, Hans-Andreas Engel, Emmanuel I. Rashba, and Bertrand I. Halperin \\ Department of Physics, Harvard University, Cambridge, Massachusetts 02138, USA \\ (Received 25 March 2007; revised manuscript received 14 May 2007; published 12 June 2007)
}

\begin{abstract}
Spin polarization may be generated far from the boundaries of a sample by nonlinear effects of an electric current, in the presence of "extrinsic" spin-orbit interactions, even when such generation is forbidden in the linear regime. We present a Corbino model where spin accumulation results from a combination of current gradients, nonlinearity, and cubic anisotropy. Further, we show that even with isotropic conductivity, nonlinear effects in a low-symmetry sidearm geometry can generate spin polarization far away from boundaries. Finally, we find that drift from the boundaries dominates spin polarization patterns observed in recent experiments on GaAs by Sih et al. [Phys. Rev. Lett. 97, 096605 (2006)].
\end{abstract}

DOI: 10.1103/PhysRevB.75.241202

PACS number(s): 72.25.Dc, 71.70.Ej

Spin polarization can be generated and manipulated in semiconductors by means of electric fields and spin-orbit coupling. A prominent example is the spin Hall effect, ${ }^{1-10}$ where a homogeneous electric current passing through a sample induces spin polarization $\mathbf{s}(\mathbf{r})$ near lateral edges, with opposite polarization at opposite edges. For rectangular homogeneous samples, this spin polarization falls off exponentially when moving away from the boundary on the length scale of the spin diffusion length $L_{\mathrm{s}}$, so for samples of size $L>L_{\mathrm{s}}$ no spin polarization due to the spin Hall effect is expected far away from the edges (on scale $L$ ). However, in recent experiments on low-symmetry samples, Sih et al. ${ }^{11}$ observed polarized spins away from the edges of a GaAs sample subjected to an electric current and concluded that there are transport effects beyond the simplest spin Hall effect near edges.

In existing analytical theories of the spin Hall effect, spin polarization has been considered in the linear transport regime for small electric field $\mathbf{E}$. Here we show that in this regime, spin generation away from boundaries of homogeneous samples is forbidden for extrinsic spin-orbit interactions. By considering nonlinearities in charge transport, however, we find a new mechanism of generating electron spins by an inhomogeneous electric current. This nonlinear regime is of practical importance, because experiments are often performed in a range of electric fields with nonlinear currentvoltage characteristics. ${ }^{3,12}$ We then present an analytically solvable Corbino model in which the bulk spin generation in a radially symmetric sample geometry is due to the nonlinearity and the anisotropy of conductivity tensor. Finally, for the case of nonlinear but isotropic conductivity, we numerically solve the charge transport and spin drift-diffusion equations for a sidearm geometry used in Ref. 11 . The patterns of spin accumulation we find strongly resemble experimental findings. We establish the existence of two contributions to the spin polarization: the first contribution is generated at the boundaries and then drifts large distances (while diffusing farther away from the boundaries), and the second one is generated away from the boundaries. While we find that in recent experiments the first of these dominated, we propose setups that should allow unambiguous observation of the spins generated in the bulk.

Spin generation. We consider a diffusive system with a weak extrinsic spin-orbit coupling and typical system size $L$, and consider spins quantized along $\hat{\mathbf{z}}$. The spin current contains drift and diffusion contributions. Additionally, electrical current induces a spin Hall current $\mathbf{j}_{\mathrm{SH}}^{z}$, which acts as a source for the spin polarization $s_{z}(\mathbf{r})$ with a generation rate $\Gamma_{z}=-\operatorname{div} \mathbf{j}_{\mathrm{SH}}^{z}$. For length scales much larger than the mean free path $\ell$, spin-orbit processes (e.g., Dyakonov-Perel or Elliott-Yafet mechanisms) lead to a finite spin lifetime $\tau_{\mathrm{s}}$. Together with a spin diffusion coefficient $D_{\mathrm{s}}$, the spin lifetime defines a spin diffusion length $L_{\mathrm{s}}=\sqrt{D_{\mathrm{s}} \tau_{\mathrm{s}}}$. To analyze the spin polarization away from boundaries, we consider the regime $\ell \ll L_{\mathrm{s}}<L$. On length scales large compared to $\ell$ and in the absence of an external magnetic field, the spin density $s_{z}(\mathbf{r})$ obeys the drift-diffusion equation

$$
\dot{s}_{z}=\operatorname{div}\left(D_{\mathrm{s}} \nabla s_{z}\right)-\operatorname{div}\left(\mathbf{v}_{\mathrm{dr}} s_{z}\right)+\Gamma_{z}-\frac{s_{z}}{\tau_{\mathrm{s}}},
$$

where the drift velocity $\mathbf{v}_{\mathrm{dr}}$ is proportional to the local electric current density. This introduces yet another length scale, the drift length $L_{\mathrm{dr}}=v_{\mathrm{dr}} \tau_{\mathrm{s}}$.

In the linear transport regime, one can evaluate $\Gamma_{z}$ by writing the spin current induced by the electric field as $\mathbf{j}_{\mathrm{SH}}^{z}=\hat{\sigma}^{\mathrm{SH}} \mathbf{E}$, with a spin Hall conductivity tensor $\hat{\sigma}^{\mathrm{SH}}$ that does not depend on E. Assuming noninteracting electrons, in the absence of intrinsic spin-orbit coupling, one can independently consider the spin species with opposite polarization and then relate the spin Hall effect to the anomalous Hall effect. ${ }^{7}$ In particular, at magnetic field $B=0$, we find $d \hat{\sigma}^{\mathrm{SH}} / d \varepsilon_{\mathrm{F}}=(\hbar / 2 \mu e) d \hat{\sigma}^{\mathrm{AH}} /\left.d B\right|_{B=0}$, with $\hat{\sigma}^{\mathrm{AH}}=\frac{1}{2}[\hat{\sigma}(B)$ $-\hat{\sigma}(-B)]$ and where $\hat{\sigma}(B)$ is magnetic field-dependent charge conductivity, $\varepsilon_{\mathrm{F}}$ is the Fermi energy, $\mu$ is the magnetic moment, and $e$ is the electron charge. From the Onsager relation for $\hat{\sigma}(B)$, we see that $\hat{\sigma}^{\mathrm{SH}}$ must be antisymmetric. Using this property for a homogeneous two- or three-dimensional system, we find

$$
\Gamma_{z}=-\frac{1}{2} \sum_{i j k} \sigma_{i j}^{\mathrm{SH}} \epsilon_{i j k}(\nabla \times \mathbf{E})_{k}=0 .
$$

Although the derivatives of $\mathbf{E}(\mathbf{r})\left(\propto \frac{1}{L}\right)$ are possible far away from the boundary, $\boldsymbol{\nabla} \times \mathbf{E}$ vanishes. 
We have found above that, away from boundaries, extrinsic spin generation is forbidden in the linear transport regime in homogeneous samples. As we will shortly see, nonlinear effects can lead to such bulk spin generation. We will restrict ourselves to the case of extrinsic spin current generated by spin-dependent impurity scattering; it is given by $\mathbf{j}_{\mathrm{SH}}^{\mathrm{z}}$ $=\hat{\mathbf{z}} \times\left(\frac{\gamma}{2 e} \mathbf{J}_{\mathrm{c}}-2 \lambda n \frac{e}{\hbar} \mathbf{E}\right),{ }^{7}$ where the two terms correspond to the skew-scattering and side-jump contributions, respectively. Here, $\mathbf{J}_{c}=\hat{\sigma} \mathbf{E}$ is the charge current, but now we allow the conductivity tensor to be a function of the electric field. Further, $\lambda$ is a material-dependent spin-orbit coupling constant and the skewness $\gamma$ is of order $\lambda$ but it also depends on the properties of scatterers and on electron distribution function. The side-jump part of the current does not contribute to $\Gamma_{z}$, and we obtain

$$
\Gamma_{z}=\frac{1}{2 e}(\boldsymbol{\nabla} \times \gamma \hat{\sigma} \mathbf{E})_{z} .
$$

Corbino model. We now show how an anisotropic, nonlinear conductivity leads to spin generation away from the boundaries. In general, according to Eq. (3), inhomogeneous electric fields are required for a finite $\Gamma_{z}$. Thus, we now analyze the inhomogeneous field in a Corbino geometry, where a total current $I$ is injected at $r=a$ into an infinite two-dimensional sample. In a (001) film of crystal of full cubic symmetry, the leading nonlinearities in $\mathbf{J}_{\mathfrak{c}}(\mathbf{E})$ are

$$
J_{\mathrm{c}}^{i}=\left(\sigma+\sigma_{2} E^{2}\right) E_{i}+\sigma_{1} E_{i}^{3},
$$

where the components $i=x, y$ are taken along the principal crystal axes and we assume that $\sigma_{1,2} E^{2}(a) \ll \sigma$. Anisotropic nonlinear terms can be of considerable magnitude for manyvalley semiconductors such as $\mathrm{SiGe}$ quantum wells and also for $\mathrm{Al}_{x} \mathrm{Ga}_{1-x} \mathrm{As}$ quantum wells with $x$ close to the directindirect gap transition. We first solve for the electrostatic potential $\Phi(\mathbf{r})$; in polar coordinates, it will be of the form $\Sigma_{\mathrm{m}} \Phi_{\mathrm{m}}(r) \cos 4 \mathrm{~m} \varphi$. The term $\Phi_{0}(r)$ will not contribute to $\Gamma_{z}$ [Eq. (3)], so we consider the next lowest harmonic $\Phi_{1}(r) \cos 4 \varphi$. Because div $\mathbf{J}_{\mathrm{c}}=0$, we see that

$$
\frac{d^{2} \Phi_{1}(r)}{d r^{2}}+\frac{1}{r} \frac{d \Phi_{1}(r)}{d r}-\frac{16}{r^{2}} \Phi_{1}(r)+\frac{3 \sigma_{1}}{2 \sigma r^{4}}\left(\frac{I}{2 \pi \sigma}\right)^{3}=0 .
$$

Requiring that $\Phi_{1}(r \rightarrow \infty)=0$, we obtain

$$
\Phi_{1}(r)=\frac{\sigma_{1}}{8 r^{2}}\left(\frac{I}{2 \pi \sigma}\right)^{3} .
$$

The spin generation rate is then

$$
\Gamma_{z}(r, \varphi)=\frac{3 \gamma}{4 e} \sigma_{1}\left(\frac{I}{2 \pi \sigma}\right)^{3} \frac{\sin 4 \varphi}{r^{4}} .
$$

So indeed, the combined anisotropy and nonlinearity of conductivity lead to a spin generation, which, for $r \gg L_{\mathrm{s}}$, results in the spin density $s_{z}(r, \varphi)=\Gamma_{z}(r, \varphi) \tau_{\mathrm{s}} \propto I^{3} \sin (4 \varphi) / r^{4}$. We emphasize that this polarization falls off only as a power law and that it consists of four sectors of up spins separated by four sectors of down spins.

Sample with sidearm. We now analyze the out-of-plane spin polarization $s_{z}(\mathbf{r})$ in systems with isotropic (nonlinear)

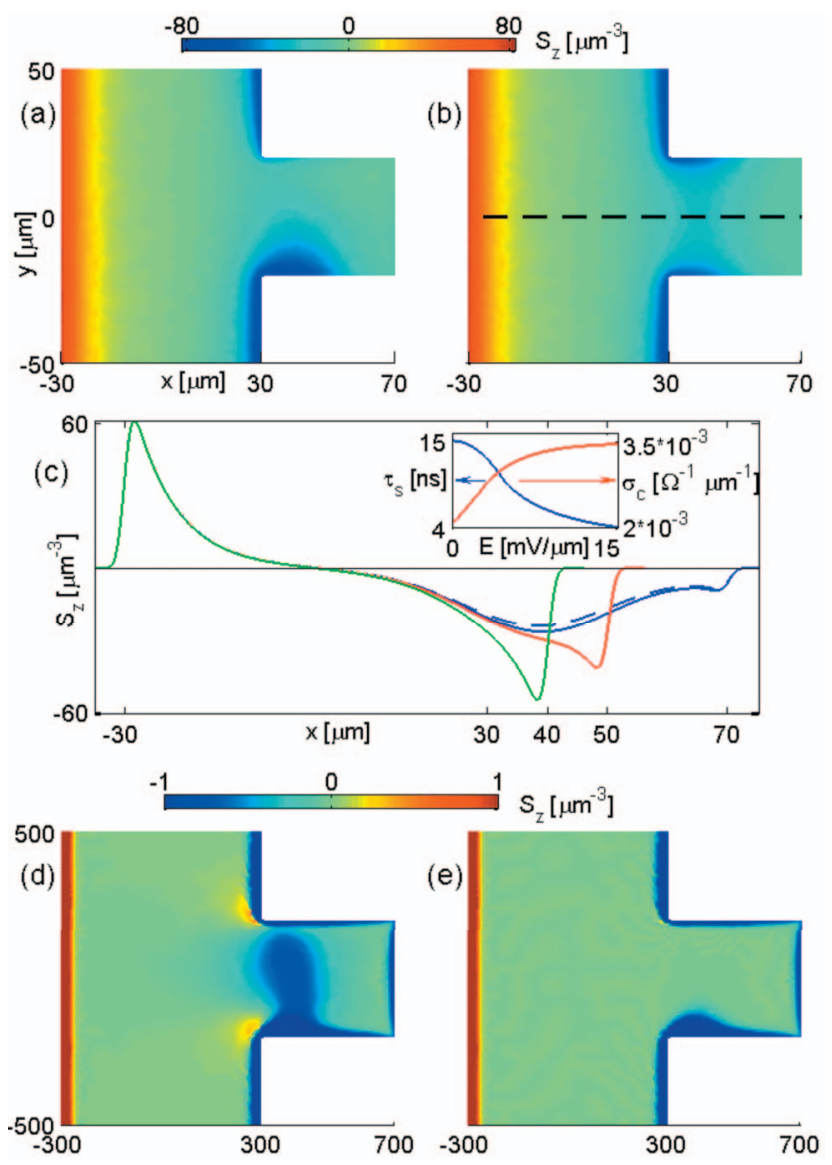

FIG. 1. (Color) Simulated spin accumulation $s_{z}(\mathbf{r})$ for experimental geometry and parameters of Ref. 11. Electron flow is from bottom to top in the main channel and spills over into the sidearm. Distances are measured in microns, and the electric field in the main channel is $9.5 \mathrm{mV} / \mu \mathrm{m}$. (a) Numerical solution of spin driftdiffusion equation [Eq. (1)] for a $40-\mu \mathrm{m}$ sidearm in the dc regime. (b) Symmetrized spin accumulation $\frac{1}{2}\left[s_{z}(V)-s_{z}(-V)\right]$. (c) Spin polarization $s_{z}(x, y)$ (convoluted with a Gaussian with standard deviation of $1 \mu \mathrm{m}$ that is associated with laser spot) for $y=0$ [along the dashed line in (b)] and for sidearms of depth $10 \mu \mathrm{m}$ (green), $20 \mu \mathrm{m}$ (red), and $40 \mu \mathrm{m}$ (blue) is in good agreement with the experimental data in Fig. 1(c) of Ref. 11. The dashed line shows a simulation without bulk spin generation $\left(\Gamma_{z}=0\right)$. The inset shows spin relaxation time $\tau_{\mathrm{s}}(E)$ and charge conductivity $\sigma(E)$ as used in our simulation. Panels (d) and (e) show $s_{z}(r)$ on a different color scale for a sample larger by a factor of $\alpha=10$. In (e), the spin generation rate $\Gamma_{z}$ is neglected.

conductivity but with less symmetric geometries. We consider unstrained GaAs samples with a sidearm geometry (see Fig. 1) that were used in experiments by Sih et al. ${ }^{11}$ The spin Hall effect in such samples is believed to be primarily of extrinsic origin., 3,7, We note that in samples where the extrinsic spin Hall effect was observed ${ }^{3,11}$ the charge conductivity $\sigma(E)$ was found to be an increasing function of field. ${ }^{3,12}$ In a sample with sidearm, subjected to an electric field, electrons flow from the bottom to the top of the main channel, some of them entering into the sidearm; the gradient of the field magnitude $E(\mathbf{r})$ becomes large near the entrance of the arm, and a field-dependent conductivity will then lead 
to spin generation across this region. Furthermore, due to the spin Hall effect, spins are also generated near the sample edges and can then diffuse and drift along the electric field into the center of the sidearm-below, we find that this latter mechanism dominates the experimental observations.

We choose realistic values of the parameters as follows. Electron density $n=3 \times 10^{16} \mathrm{~cm}^{-3}$ and sample dimensions are taken from Ref. 11. Further, we assume that the sample is homogeneous and the spatial dependence of $\sigma, \tau_{\mathrm{s}}$, and $D_{\mathrm{s}}$ is controlled by the local field $E(\mathbf{r})$. Low-field conductivity $\sigma(E)$ for GaAs samples was obtained from unpublished data, ${ }^{12}$ and for higher fields we took the $E$ dependence of the conductivity measured for $\operatorname{In}_{0.07} \mathrm{Ga}_{0.93} \mathrm{As}$ in Ref. 3 as a guideline (there, it increased by a factor of 2 as the field increased from 0 to $20 \mathrm{mV} / \mu \mathrm{m})$. The spin relaxation time $\tau_{s}(E)$ was loosely based on the experimental data taken at two points away from the boundaries for a range of electric fields; ${ }^{12}$ we show our assumed $\sigma(E)$ and $\tau_{s}(E)$ in Fig. 1(c). Furthermore, it was found in Ref. 3 that the spin diffusion length $L_{s}$ was field independent within error bars; thus, we take $D_{s}(E)=L_{s}^{2} / \tau_{s}(E)$ with constant $L_{s}$. We chose $L_{s}=7 \mu \mathrm{m}$ found from the best fit to the data of Ref. 11 for the main channel. We take the spin-orbit coupling constant $\lambda=5.3 \AA^{2}$ and estimate the skewness $\gamma=1 / 700 .^{7,13}$ Finally, we assume that $\gamma$ is constant, the conductivity is isotropic, and that its position dependence is only through the magnitude $E$ of the local field; Eq. (3) then simplifies to

$$
\Gamma_{z}=\frac{\gamma}{2 e} \frac{d \sigma}{d E}(\nabla E \times \mathbf{E})_{z} .
$$

In our simulation, we first solve for the electrostatic potential $\Phi(\mathbf{r})$ for an applied dc voltage and determine the spin generation rate $\Gamma_{z}(\mathbf{r})[\mathrm{Eq} .(8)]$ and the drift velocity $\mathbf{v}_{\mathrm{dr}}(\mathbf{r})$ $=\mathbf{J}_{\mathrm{c}}(\mathbf{r}) /$ ne. Because there are no experimental indications of an excess spin relaxation at the boundaries, we consider the spin-conservation boundary condition $\hat{\mathbf{n}} \cdot\left(D_{\mathrm{s}} \nabla s_{z}-\mathbf{j}_{\mathrm{SH}}^{z}\right)=0$. This accounts for the spin generated at the boundaries due to the spin Hall effect. We then solve the spin drift-diffusion equation [Eq. (1)] and find the stationary spin polarization $s_{z}(\mathbf{r})$.

The simulated $s_{z}(\mathbf{r})$ is shown in Fig. 1(a). The most striking feature is an asymmetric spin distribution inside the sidearm: it results from spins generated at the boundaries near the corner of the lower part of the sidearm, which diffuse and then drift a distance $L_{\mathrm{dr}}=v_{\mathrm{dr}} \tau_{\mathrm{s}}$, which is much longer than $L_{\mathrm{s}}$. Conversely, near the upper part of the sidearm the spin population is very low, since spins generated at the boundary drift out of the sidearm. However, in Ref. 11 a square-wave voltage $V$ was applied for lock-in detection; the measured spin polarization $\frac{1}{2}\left[s_{z}(V)-s_{z}(-V)\right]$ then becomes symmetric, and we show the "symmetrized" polarization in Fig. 1(b). We find good agreement with experimental data [see Fig. 1(c) in Ref. 11] in particular, for the sample with a $40-\mu \mathrm{m}$ sidearm, the maximum of spin distribution is at a similar position, about $10-15 \mu \mathrm{m}$ into the sidearm. In Fig. 1(c), we show $s_{z}$ along the central section of the sidearm for different depths of the sidearm and find that for depths of 10 or $20 \mu \mathrm{m}$, the spin population is maximal at the right edge of the arm.
The simulation includes contributions from spins generated away from boundaries with rate $\Gamma_{z}[$ Eq. (8)]. For comparison, we show the spin polarization for $\Gamma_{z}=0$ [see dashed line in Fig. 1(c)]. We find that the absence of $\Gamma_{z}$ changes the resulting spin polarization by less than $15 \%$. Therefore, in the experiments of Ref. 11, the dominant contribution to the stationary spin distribution inside the sidearm comes from the drift of the spins generated at the sample boundaries. This is a consequence of drift distance $L_{\mathrm{dr}}$ being larger than the width of the sidearm.

We now discuss why intrinsic effects linear in $E$ are not expected to be important in the experiments of Ref. 11. For low-symmetry (two-dimensional) samples with $k$-linear intrinsic spin-orbit interaction, there is a well-known mechanism that produces (typically in-plane) spin polarization linear in the homogeneous electric field. ${ }^{14,15}$ In our case, the $k$-linear mechanism is not expected since the experimental samples were those of unstrained bulk GaAs. More generally, the cubic symmetry group $T_{d}$ of GaAs does not allow for $s_{z}$ linear in $\mathbf{E}$ up to a first order in a gradient expansion of $\mathbf{E}$ (r) (up to a second order, if $z$ is a principal crystallographic axis). Therefore, we attribute, in agreement with Ref. 3, the observations to the extrinsic effect.

Identifying spin generation away from the boundaries. In order to distinguish spins generated in the bulk from those originating at the boundaries, one needs a setup where both $L_{\mathrm{s}}$ and $L_{\mathrm{dr}}$ are smaller than the width of the sidearm, $L$. While $L_{\mathrm{s}}<L$ was satisfied in the experiments of Ref. 11 and in the simulations above, $L_{\mathrm{dr}}<L$ was not. The inequality can be achieved (i) by reducing $L_{\mathrm{dr}}$ with a smaller applied field (and, therefore, smaller drift velocity), (ii) by reducing $L_{\mathrm{dr}}$ by pulsing the applied fields and thus allowing drift only for a shorter time, (iii) by using an alternating sequence of pulsed electric fields, which can largely cancel the drift and the spin generation at the boundaries, or (iv) by increasing sample dimensions $L$. This exponentially suppresses the contributions from the boundaries (as long as $L_{\mathrm{s}}$ and $L_{\mathrm{dr}}$ are sufficiently short), making the relative contribution of the generation away from boundaries more sizable. However, this also suppresses the absolute value of spin polarization, as we discuss now.

For approach (i), a smaller drift velocity is achieved by choosing a sufficiently small electric field, so that $v_{\mathrm{dr}} \ll L / \tau_{\mathrm{s}}$. Because of the scaling $\Gamma_{z} \propto \sigma^{\prime}(E) E^{2} / L$, with $\sigma^{\prime}(E)=d \sigma / d E$, small drift velocities also imply that spin polarization due to generation away from boundaries is weak. (ii) When the electric field is only applied in pulses of duration $t_{\mathrm{p}}$, which are short compared to $\tau_{\mathrm{s}}$, the drift length is reduced to $L_{\mathrm{dr}}$ $=t_{\mathrm{p}} v_{\mathrm{dr}}$. Also, the pulses must be at least $\tau_{\mathrm{s}}$ apart, and thus the average spin polarization signal is reduced from the dc case by a factor smaller than $t_{\mathrm{p}} / \tau_{\mathrm{s}}$. For example, applying electric field pulses with $t_{\mathrm{p}}=1 \mathrm{~ns}$ and separation time $20 \mathrm{~ns}$, for the parameters used in Fig. 1, spin generation away from boundaries could be observed with a time-averaged value of $s_{z}$ $\lesssim 0.2 \mu \mathrm{m}^{-3}$. Another approach (iii) is to use alternating pulses of large positive $E$ for duration $t_{1}$ and negative $E$ of smaller magnitude for a longer time $t_{2}$, such that the timeaveraged electric current is zero. Then, if the period $t_{1}+t_{2}$ is smaller than both $\tau_{\mathrm{s}}$ and $L / v_{\mathrm{dr}}$, the effects of spin drift should be largely canceled. Also, during $t_{1}$ and $t_{2}$ equal amounts of 
spin polarization are generated at the boundary, but with opposite signs, which leads to a cancellation of the contribution of spins generated at the boundaries. In contrast, since the bulk spin generation $\Gamma_{z}$ is nonlinear, it can have a nonzero time average. The effectiveness of this scheme will depend on the strength of nonlinearities involved.

Note that in approaches (i)-(iii), diffusion of spins generated at the boundaries may still dominate $s_{z}(\mathbf{r})$ in the bulk. For example, in simulations with the geometry of Fig. 1 but for smaller fields, the bulk $s_{z}$ still contains a large relative contribution from spins generated at the boundaries. To eliminate such a diffusion effect, one could use approach (ii) in a pump-probe scheme, where the spin polarization is detected shortly after the pulse, providing a direct measurement of $\Gamma_{z}(\mathbf{r})$.

The most straightforward way to reveal spin generation away from boundaries is to increase the sample's linear dimensions by a factor of $\alpha$ large enough so that the sidearm opening exceeds the drift length $L_{\mathrm{dr}}$, while keeping the average electric field in the main channel fixed (iv). This reduces $\Gamma_{z}$ and hence the bulk spin polarization by a factor of $\alpha$. The signal to noise should not decrease as the signal can be averaged over an area that is larger by a factor of $\alpha^{2}$, thus reducing the noise by $\alpha$. Figures 1(d) and 1(e) demonstrate spin populations in a sample 10 times larger than the sample of Fig. 1. A noteworthy feature is found in the vicinity of the corners: the spin population there is of the opposite sign than the population on the adjacent boundary. For comparison, we show in Fig. 1(e) the spin polarization for $\Gamma_{z}=0$. No spin polarization is found in the central part of the sidearm; thus, the polarization in the central part of Fig. 1(d) indeed results from spins generated away from boundaries.
In conclusion, we have shown that, in a linear transport regime, the (extrinsic) generation of spin polarization away from boundaries is forbidden. However, in nonlinear transport, spins can be generated away from boundaries and we analyze such generation resulting from spin Hall currents due to the "extrinsic" skew scattering mechanism. For an anisotropic nonlinear charge conductivity tensor, we analytically evaluate the spin generation in a Corbino geometry. We also simulate the spin polarization in a sidearm geometry using isotropic nonlinear conductivity and field-dependent spin lifetimes, appropriate to recent experiments. We find that the spin accumulations in the experiments of Ref. 11 were primarily due to drift of spins generated at the boundaries, but we suggest other setups, where drift should be relatively unimportant and nonlinear spin generation away from boundaries should be observable.

In a recent work, Stern et al. ${ }^{12,16}$ have independently shown that drift alone can account for observations of Ref. 11. Golizadeh-Mojarad and Datta ${ }^{17}$ have obtained spin polarizations qualitatively similar to those seen in experiments ${ }^{11}$ using a model with intrinsic (Rashba) spin-orbit coupling. However, their calculation assumes that $\ell \approx L_{\mathrm{s}}$. Pershin and Di Ventra ${ }^{18}$ considered rectangular samples with inhomogeneous charge density $n$, which leads to spin generation linear in the electric field, assuming that $\sigma \propto n$ [cf. Eq. (3)].

We thank D. D. Awschalom for enlightening discussions and for providing us with unpublished data and N. P. Stern for discussing the results. This work was supported in part by NSF Grant Nos. DMR05-41988 and PHY01-17795 and by the Harvard Center for Nanonscale Systems.
${ }^{1}$ M. I. Dyakonov and V. I. Perel, Phys. Lett. 35A, 459 (1971).

${ }^{2}$ J. E. Hirsch, Phys. Rev. Lett. 83, 1834 (1999).

${ }^{3}$ Y. K. Kato, R. C. Myers, A. C. Gossard, and D. D. Awschalom, Science 306, 1910 (2004).

${ }^{4}$ S. Murakami, N. Nagaosa, and S.-C. Zhang, Science 301, 1348 (2003).

${ }^{5}$ J. Sinova, D. Culcer, Q. Niu, N. A. Sinitsyn, T. Jungwirth, and A. H. MacDonald, Phys. Rev. Lett. 92, 126603 (2004).

${ }^{6}$ J. Wunderlich, B. Kaestner, J. Sinova, and T. Jungwirth, Phys. Rev. Lett. 94, 047204 (2005).

${ }^{7}$ H.-A. Engel, B. I. Halperin, and E. I. Rashba, Phys. Rev. Lett. 95, 166605 (2005).

${ }^{8}$ W.-K. Tse and S. Das Sarma, Phys. Rev. Lett. 96, 056601 (2006).

${ }^{9}$ J. Shi, P. Zhang, D. Xiao, and Q. Niu, Phys. Rev. Lett. 96, 076604 (2006)

${ }^{10}$ For theory reviews, see H.-A. Engel, E. I. Rashba, and B. I. Halperin, arXiv:cond-mat/0603306 (unpublished); J. Schlie- mann, Int. J. Mod. Phys. B 20, 1015 (2006), and references therein.

${ }^{11}$ V. Sih, W. H. Lau, R. C. Myers, V. R. Horowitz, A. C. Gossard, and D. D. Awschalom, Phys. Rev. Lett. 97, 096605 (2006).

${ }^{12}$ D. D. Awschalom (private communication).

${ }^{13}$ This value of $\gamma$ is $20 \%$ larger than the one in Ref. 7, because we account for a typo in Eq. (1A-107) in J. W. Motz, H. Olsen, and H. W. Koch, Rev. Mod. Phys. 36, 881 (1964); this correction does not affect the conclusions of Ref. 7.

${ }^{14}$ V. M. Edelstein, Solid State Commun. 73, 233 (1990).

${ }^{15}$ A. G Aronov, Y. B. Lyanda-Geller, and G. E. Pikus, JETP 73, 537 (1993).

${ }^{16}$ N. P. Stern et al. (unpublished).

${ }^{17}$ R. Golizadeh-Mojarad and S. Datta, arXiv:cond-mat/0703280 (unpublished).

${ }^{18}$ Y. Pershin and M. Di Ventra, arXiv:cond-mat/0703310 (unpublished). 